\title{
Schützt Ibuprofen vor Blasenkrebs?
}

\author{
Wer über zehn Jahre lang Ibuprofen einnimmt, hat einer Studie zufolge \\ möglicherweise ein niedrigeres Blasenkrebsrisiko. Für den protektiven Effekt \\ könnten Genvarianten im NSAR-Metabolismus verantwortlich sein.
}

\begin{abstract}
E in Zusammenhang zwischen der Einnahme von nicht steroidalen Antirheumatika (NSAR) und einem geringeren Blasenkrebsrisiko hatte sich bereits in einzelnen früheren Studien angedeutet. Diesen Hinweisen gingen US-amerikanische Wissendchaftler in einer populationsbasierten Fall-Kontroll-Studie nach. Die Wissenschaftler nahmen 1.171 Personen zwischen 30 und 79 Jahren mit neu diagnostiziertem Blasenkrebs und 1.418 Kontrollpersonen in die Studie auf, befragten sie unter anderem nach ihrem Schmerzmittelgebrauch und untersuchten Gene, die als Kandidaten für den NSARMetabolismus gelten. $\mathrm{Zu}$ den eingenommenen Schmerzmitteln zählten Paracetamol, ASS, die nicht selektiven NSAR Ibuprofen und Naproxen sowie die COX2-Inhibitoren Celecoxib, Rofecoxib und Valdecoxib.
\end{abstract}

Probanden, die regelmäßig nicht selektive NSAR (ohne ASS) eingenommen hatten, zeigten nach fünf bis neun Jahren ein um $20 \%$ (nicht signifikant) niedrigeres Blasenkrebsrisiko als solche, die nie mit entsprechenden Schmerzmitteln behandelt wurden. Dabei ergab sich eine signifikante Abhängigkeit von Einnahmedauer und Dosierung. Ab einer Medikationsdauer von zehn Jahren sank das Risiko um $40 \%$. Am deutlichsten zeigte sich dieser Zusammenhang in der Einzelauswertung für Ibuprofen. Die Genomanalyse legte allerdings nahe, dass dieser Vorteil auf Patienten mit bestimmten Nukleotidpolymorphismen (SNP) im CYP3A-Locus beschränkt war. Ihr Risiko war gegenüber Probanden mit homozygotem WildtypCYP3A, die nie Ibuprofen eingenommen hatten, je nach Genvariante um $50 \%$ beziehungsweise $70 \%$ niedriger.
Bei Patienten, die selektive COX-2Hemmer einnahmen war das Risiko, einen Blasenkrebs zu entwickeln, im Durchschnitt um $30 \%$ erhöht und stieg mit der Dauer der regelmäßigen Einnahme von Celecoxib, nach drei Jahren etwa auf das Doppelte der Kontrollgruppe.

Fazit: Die Studie bestätigt Ergebnisse früherer Untersuchungen zur protektiven Wirkung von NSAR auf die Entwicklung eines Blasenkrebses. Von Celecoxib hätte man sich wegen seiner Eigenschaft als selektiver COX-2-Hemmer ähnliche Ergebnisse erwartet. Die Studie gibt neue Hinweise darauf, dass dem protektiven Effekt von Ibuprofen ein Genpolymorphismus in CYP3A zugrunde liegt. Würde infolge einer geringeren Genexpression Ibuprofen langsamer verstoffwechselt, stünde letztlich mehr aktiver Wirkstoff mit antikanzerogener Wirkung zur Verfügung, so eine Erklärung. Dr.Christine Starostzik

Baris $D$ et al. Nonsteroidal anti-inflammatory drugs and other analgesic use and bladder cancer in northern New England. Int J Cancer 2013; 132: 162-73

\section{ED: Testosteron zusätzlich zu PDE-5-Hemmern?}

\author{
Verstärkt eine zusätzliche Testosteronzufuhr die Erektionsfähigkeit bei \\ Männern mit niedrigen Hormonspiegeln, die Sildenafil einnehmen? Einer \\ aktuellen Studie zufolge lohnt die Mühe nicht.
}

n einer US-amerikanischen randomisierten, placebokontrollierten DoppelblindStudie wurde untersucht, ob die transdermale Zufuhr von Testosteron zusätzlich zu einer bestehenden Sildenafil-Therapie die erektile Dysfunktion (ED) bei Männern mit einem Gesamttestosteron < 11,45 nmol/l und einem freien Testosteron $<173,35 \mathrm{pmol} / \mathrm{l}$ weiter verbessern kann. 70 der im Vorfeld bedarfsgerecht auf eine optimale Sildenafildosis zwischen 25 und $100 \mathrm{mg}$ eingestellten Probanden trugen zusätzlich 14 Wochen lang täglich $10 \mathrm{~g}$ Testosterongel $1 \%$ auf, 70 Kontrollpersonen unter Sildenafil erhielten ein Hormonplacebo. Die Testosteronspiegel, die zu Beginn im Durchschnitt bei 8,61 nmol/1 beziehungsweise $8,81 \mathrm{nmol} / \mathrm{l}$ lagen und bereits unter Sildenafil um etwa durch- schnittlich 3,5 nmol/l anstiegen, wurden zwei Wochen nach der Randomisierung nochmals überprüft und die Dosis so angepasst, dass sich die Serumwerte zwischen 17,35 und 34,7 nmol/l einpendelten. Der mittlere Testosteronwert stieg in der Testosterongruppe auf 22,52 nmol/l.

Die zu Beginn der Studie ähnlichen IIEF-Scores besserten sich in beiden Gruppen durch die Einnahme von Sildenafil ähnlich (von 12,1 auf 19,8), aber der erhoffte Effekt auf eine bessere Erektionsfähigkeit durch zusätzliche Testosteronzufuhr blieb aus. Auch auf andere Bereiche sexueller Funktion wie etwa der erfolgreichen vaginalen Penetration, der Ejakulation oder der Gesamtzufriedenheit mit dem Sexualleben hatte die Hormongabe keinen Einfluss. Auch diejenigen, die besonders schlecht auf Sildenafil angesprochen hatten, oder Männer mit einem Ausgangstestosteronwert $<8,68 \mathrm{nmol} / \mathrm{l}$ hatten keine weiteren Vorteile durch kombiniertes transdermales Testosteron.

Fazit: Das Ausbleiben eines Effekts auf die ED durch die zusätzliche Gabe von Testosteron überrascht in Anbetracht der Studienlage zunächst, so die Autoren. Sie betonen, dass die Ergebnisse auf keinen Fall so interpretiert werden dürften, dass eine Testosteronbehandlung bei Männern mit niedrigem Hormonspiegel generell keinen Nutzen habe. Allerdings zeige die Studie, dass die heute bereits weit verbreitete routinemäßige Testosterongabe zusätzlich zu PDE-5-Inhibitoren die Erektionsfähigkeit bei Männern mit niedrigen Testosteronspiegeln und ED nicht weiter verbessert.

Dr. Christine Starostzik

Spitzer M et al. Effect of Testosterone Replacement on Response to Sildenafil Citrate in Men With Erectile Dysfunction: A Parallel, Randomized Trial. Ann Intern Med 2012; 157: 681-91 